\title{
Análisis del desarrollo fonológico en sujetos malagueños
}

\author{
MARIA JOSE GONZALEZ \\ Universidad de Málaga
}

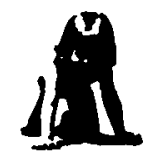

\section{Resumen}

El presente estudio intenta analizar, desde una perspectiva psicolinguística, el desarrollo fonológico de niños castellanoparlantes de la ciudad de Málaga, en función de variables como el sexo, el nivel sociocultural y edad de los sujetos, atendiendo, por otra parte, a la influencia de caracteristicas dialectales del babla andaluza.

Tras la elaboración y administración de una prueba de screening, en condiciones de imitación, se registraron las producciones correctas y distintos procesos de simplificación del babla (sustitución, relativos a la estructura de la silaba y asimilatorios), no encontrándose diferencias significativas con respecto al sexo, aunque si en cuanto al nivel sociocultural y a la edad de los sujetos.

Cabe destacar que la influencia del modelo lingüistico andaluz podría enmascarar los patrones evolutivos del desarrollo fonológico infantil. Sin embargo, el hecho de que los sujetos malagueños presenten porcentajes bajos en pronunciación correcta en edades avanzadas, en algunas categorias de fonemas, es debido a la influencia de variantes dialectales, más a que presenten patrones evolutivos más tardios en cuanto a su capacidad de representación fonológica.

Palabras clave: Desarrollo fonológico. Perspectiva Psicolinguística. Capacidad de representación fonológica. Variantes dialectales andaluzas. Sexo. Nivel sociocultural. Edad.

\section{Analysis of the phonological development of children from Málaga}

\begin{abstract}
The present study tries to analyse from a psycholinguistic perspective the phonological development of spanish speaking children from the city of Málaga, as a function of variables such as sex, sociocultural level and the child's age. The influence of the dialectical characteristics of Andalusian speech were also studied.

After the elaboration and administration of a screening test under conditions of imitation, the correct articulations and different processes of simplification of speech were recorded. No significant differences uere obtained for sex, though differences were found with regards to sociocultural level and cbild age.

It is necessary to underline that the influence of Andalusian linguistic patterns might mask the developmental patterns of child phonology. However, the fact that in some phoneme categories subjects from Málaga present low percentages in correct articulation at advanced ages is due to the influence of the dialectal variants rather than to a slower development in their ability for phonological representation.
\end{abstract}

Key words: Phonological development. Psycholinguistic perspective. Phonological representation ability. Andalusian dialectal variant. Sex. Sociocultural level. Age.

Agradecimientos: La investigación fue realizada bajo la dirección de la Profesora Titular del Departamento de Psicología de la Universidad de Málaga, Rosa Ana Clemente Estevan, y con la colaboraciộn de Sonia Codes Requena, alumna del mismo.

Dirección del autor: Dpto. de Psicología. Edificio Rectorado. El Ejido, s/n. Universidad de Málaga. 29013 Málaga.

Original recibido: enero de 1989, Revisión recibida: julio 1989. Aceptado: julio 1989. 


\section{INTRODUCCION}

La necesidad de llevar a cabo estudios que evalúen patrones evolutivos del habla, y que postulen reglas o procesos de adquisición fonológica, se hace cada vez más patente, a raíz de la detección de los llamados trastornos fonológicos, y su influencia en el aprendizaje lectoescritor (Alegría, 1985; Stanovich, 1986, etc.)

Los primeros y únicos trabajos realizados en habla castellana al respecto (Serra, 1979 y Bosch, 1982) ofrecen información sobre el repertorio de fonemas que los niños tienen adquirido y los errores cometidos en las distintas clases de fonemas según la edad de los sujetos.

Serra (1979) en un estudio transversal, analizó la adquisición fonológica de niños catalanes, de edades comprendidas entre los 3 y 7 años, pretendiendo analizar cuantitativamente los resultados conseguidos por los sujetos al repetir una lista de fonemas simples y compuestos. Estableció un orden de adquisición de fonemas, en función de los porcentajes de error que estos presentaban. Así, las nasales se adquieren antes que las oclusivas y fricativas, y posteriormente a éstas, las vibrantes y líquidas. De esta forma, el porcentaje de error articulatorio va disminuyendo con la edad, obteniéndose una secuencia de adquisición de los sonidos en orden creciente de dificultad. A partir de los 5 años se podría considerar adquirido el sistema fonológico, ya que no se encuentran porcentajes de errores elevados en las edades superiores. Por otra parte, clasificó cualitativamente los errores articulatorios en simples, múltiples y generalizados. Los primeros eran aquellos que se presentaban de forma aislada y estable en distintos contextos. Los segundos se consideraban como tales, cuando existían varios errores de tipo simple, y los últimos eran aquellos en los que se evidenciaba una falta de categorización de lugar y de modo de producción.

Más tarde, siguiendo a Ingram $(1974,1976)$, Bosch $(1982,1983)$ presenta una prueba para evaluar el desarrollo fonológico en sujetos entre los 3 y 7.11 años de edad, en la cual se ha basado el presente estudio. Entre otros resultados importantes, Bosch destaca que entre los 3 y 4 años de edad se produce el final de la primera etapa de adquisición fonológica. A los 4 años se consideraban dominados todos los elementos fónicos que aparecen en palabras de estructura simple. A los 6, se evidencia el final de la segunda etapa, y a los 7 existe un alto porcentaje de sujetos con errores que necesitarían atención terapéutica. A este respecto y, tras el análisis del habla desde la perspectiva psicolingüística de los procesos fonológicos de simplificación del habla, elabora un cuadro en el que resalta los procesos que intervienen más a distintas edades en los diversos grupos de fonemas, especificando el porcentaje que los utiliza (Bosch, 1983).

Posteriormente, y siguiendo en la misma línea de trabajo, en el Departamento de Psicología de la Universidad de Málaga, se pretendió analizar las producciones fonológicas de sujetos malagueños, no sólo en función de la edad, sino también del sexo y del nivel sociocultural, atendiendo además, a las características dialectales del habla malagueña.

Como ya había señalado Serra (1979) y Bosch (1982), existen características dialectales a tener en cuenta en los resultados evolutivos sobre la adquisición de los fonemas. Esto dio pie a que dadas las características del habla andaluza se intentara desenmascarar la influencia de estas variantes dialectales en el desarrollo fonológico de los sujetos malagueños, y su influencia en función del nivel sociocultural, sexo y edad cronológica. 
En un intento de explicar la adquisición fonológica infantil, los teóricos que abordan estos temas consideran como eje central de su teoría, la capacidad de representación simbólica, y en su consecuencia la capacidad imitativa del individuo. El niño elige las palabras que percibe de las emisiones adultas, y crea, gradualmente, sus propias estructuras en base a lo que el ambiente psicolinguístico le proporciona. En el proceso de adquisición fonológica, percibe, organiza y produce sonidos, consiguiendo que las representaciones que hace de las emisiones adultas se aproximen cada vez más a lo real, y que su sistema fonológico sea progresivamente como el del adulto al que imita, desapareciendo los procesos de simplificación del habla, característicos del niño en su periodo de desarrollo. Por tanto la influencia del modelo adulto es indispensable e inevitable en el desarrollo del lenguaje, y concretamente en el componente fonológico (Bandura, 1982; Ingram, 1976; Clark, 1977). Esquemáticamente se representa la teoría psicolingüística como sigue (Ingram, 1976):

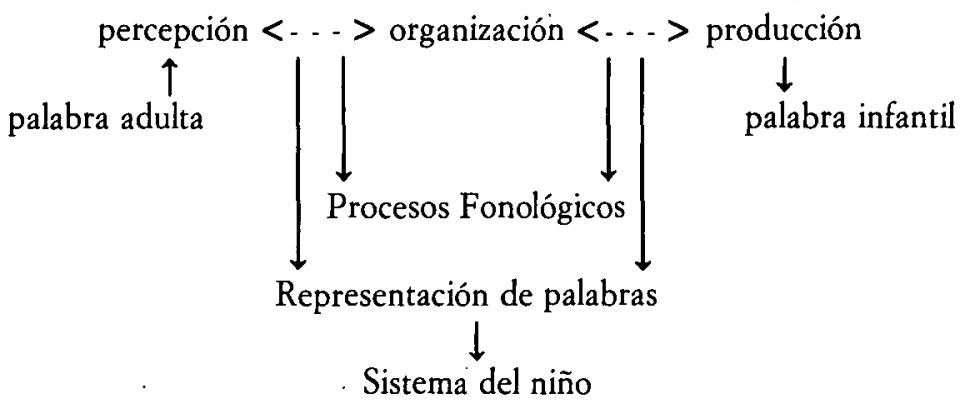

(1) repertorios de sonidos y silabas en palabras adultas

(2) repertorios de sonidos y sílabas usados por contraste

(3) repertorios de sonidos y sílabas producidos

Por todo ello, era imprescindible analizar la influencia de las variantes dialectales de nuestra lengua en la evaluación del desarrollo fonológico. Si un niño malagueño percibe sistemáticamente del modelo adulto del que aprende cómo se produce $/ \theta i j a /$ en vez de $/$ sì a/, difícilmente él imitará, producirá y será corregido, para que aprenda que aquello que sirve para sentarse se dice /si $\lambda_{a} /$ y no $/ \theta \mathrm{ija} /$. Es decir, que en nuestro afán por conocer los patrones evolutivos sobre el desarrollo fonológico, habría que considerar no sólo los aspectos lingüísticos-cognitivos del niño, sino también los sociolingüísticos, que de alguna forma interfieren el aprendizaje del lenguaje y que hace que podamos pensar en patrones evolutivos diferentes a los de los niños castellanos.

\section{METODO}

\section{Objetivos}

El objetivo principal del estudio que aquí se presenta consiste en analizar las articulaciones fonemáticas producidas por sujetos de la provincia de Málaga, tras la elaboración y administración de una prueba screening, en condiciones de 
imitación, distinguiéndose las articulaciones correctas y los distintos procesos de simplificación del habla (sustitución, relativos a la estructura de la sílaba y asimilación).

Los procesos de sustitución consisten en cambios de un fonema por otro, sin hacer referencia a los sonidos más próximos. Entre los más frecuentes cabe señalar los siguientes:

- Frontalización: sustitución de una velar $/ \mathrm{k} / \mathrm{o} / \mathrm{g} /$ por una consonante anterior. Ejemplo /gō̄o/ / $\underline{\text { boro/. }}$.

- Posteriorización: sustitución de consonantes anteriores por una velar. Ejemplo: /diente/ /biente/.

- Fricatización de oclusivas: sustitución de oclusivas por fricativas. Ejemplo: /gō̄ol / fō̄ol.

- Protusión de la lengua o ceceo: lo más común es que la /s/ se sustituya por

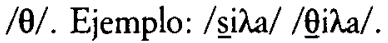

- Seseo: sustitución de / $\theta /$ por /s/. Ejemplos /taa a/ /tasa/.

- Aspiración de /s/ ante oclusivas: ejemplo /moska//mohka/.

- Pérdida de africación: la africada se convierte en fricativa, perdiendo la oclusión inicial. Ejemplo: / çaketa/ / $\underline{\text { a }}$ aketa/.

- Semiconsonantización de líquidas: las líquidas se sustituyen por $/ \mathfrak{j} / \mathrm{o} / \mathrm{w} /$. Ejemplo / $\underline{\text { ave }}$ / / jave/.

- Lateralización de liquidas vibrantes: las vibrantes $/ \mathrm{r} / \mathrm{y} / \overline{\mathrm{r}} /$ se sustituyen por /1. Ejemplo / kara/ / kala/.

- Ausencia de vibrante simple: la vibrante $/ \mathrm{r} /$ se sustituye por $/ \mathrm{d} /$. Ejemplo /kara/ /kada/.

- Aspiración de / $r /$ ante oclusivas: ejemplo: /barko//bahko/.

- etc.

Los procesos relativos a la estructura de la sílaba son aquellos que consisten en reducir las palabras a la sílaba $C V$. Entre los más frecuentes se encuentran:

- Omisión de consonantes finales, medias e iniciales: ejemplos: /tres/-/tre/, / $\underline{x}$ abon/-/abon/, /fuego/-/fueo/.

- Reducción de diptongos: ejemplo: /autobús/- /atobús/.

- Reducción de grupos consonánticos: ejemplos /kllase/-/kase/,/ /bruja/-/buja/.

Por último, los procesos de asimilación se refieren a la semejanza de un sonido por otro, dentro de la misma palabra. Tal es el caso de las asimilaciones alveolares (/libro/-/liblo/), velares (/caketa/-/kaketa/), palatales (nino/-/nijo/), etc.

\section{MATERIAL}

Como es clásico en estos temas, la prueba incluye 18 fonemas consonánticos del castellano (tabla 1), además de los 23 grupos consonánticos más frecuentes clasificados en oclusiva + líquida ( $\mathrm{br}, \mathrm{dr}, \mathrm{gr}, \mathrm{pr}, \mathrm{tr}, \mathrm{kr}, \mathrm{fr}, \mathrm{bl}, \mathrm{gl}, \mathrm{pl}, \mathrm{kl}, \mathrm{fl}$ ) y consonante + oclusiva (ls, rk, nd, nk nĉ, nt, mb, sp, st, sk, str) (1); y 7 combinaciones de vocal en diptongo ( $\mathrm{je}, \mathrm{jo}$, ja, wa, we, ei $\underline{\mathrm{iu}}$ ). Estos fonemas simples y combinados están representados en distintas posiciones dentro de la palabra (inicial, media y final), llegando a formar un total de 72 ítems. Estos ítems se reparten de la siguiente forma: uno del grupo consonántico /Ls/; dos ítems de los fonemas $/ \mathrm{f} /, / \mathrm{x} /$; africada, $/ \lambda /, / \mathrm{r} /, / \overline{\mathrm{r}} /$; tres ítems de los fonemas $/ \mathrm{s} /, / \theta / \mathrm{y} / \mathrm{L} /$; 
TABLA I

Clasificación de los fonemas consonánticos del español (Alvar y cols., 1969)

\begin{tabular}{|c|c|c|c|c|c|c|c|c|c|c|c|c|c|c|}
\hline & \multicolumn{2}{|c|}{ Bilabial } & \multicolumn{2}{|c|}{ Lab./dent. } & \multicolumn{2}{|c|}{ Lin./intdent. } & \multicolumn{2}{|c|}{ Lin./dent. } & \multicolumn{2}{|c|}{ Alveolar } & \multicolumn{2}{|c|}{ Palatal } & \multicolumn{2}{|c|}{ Velar } \\
\hline & $\mathbf{S}$ & Sn & $\mathbf{S}$ & Sn & $\mathbf{S}$ & Sn & $\mathbf{S}$ & Sn & $\mathbf{S}$ & Sn & $\mathbf{S}$ & Sn & $\mathbf{S}$ & Sn \\
\hline Oclusivas & $\mathrm{p}$ & $b$ & - & - & - & - & $\mathrm{t}$ & $\mathrm{d}$ & - & - & - & - & $\mathrm{k}$ & $\mathrm{g}$ \\
\hline Nasales & - & $\mathrm{m}$ & - & - & - & - & - & - & - & $\mathrm{n}$ & - & $\eta$ & - & - \\
\hline Fricativas & - & - & $\mathrm{f}$ & - & $\theta$ & - & - & - & s & - & - & $\mathfrak{j}$ & $\mathrm{x}$ & - \\
\hline Africada & - & - & - & - & - & - & - & - & - & - & $\hat{c}$ & - & - & - \\
\hline Líquidas laterales & - & - & - & - & - & - & - & - & - & 1 & - & $\lambda$ & - & - \\
\hline Líquidas vibrantes simples & - & - & - & - & - & - & - & - & - & $\mathrm{r}$ & - & - & - & - \\
\hline Líquidas vibrantes múltiples & - & - & - & - & - & - & - & - & - & $\overline{\mathrm{r}}$ & - & - & - & - \\
\hline
\end{tabular}


seis de nasales, siete de diptongos, diez de O.G.C.; doce de oclusivas y quince de G.C.L. El número total de palabras, representativas del habla infantil y que incluyen estos ítems fue de 40.

\section{MUESTRA}

Se les administró la prueba a un total de 198 sujetos malagueños, procedentes de escuelas públicas y privadas de habla castellana, con niveles intelectuales normales y sin problemas de tipo orgánico. Los sujetos fueron divididos en función del sexo, nivel sociocultural y edad cronológica, tal como lo expresa la tabla 2.

\section{TABLA II}

Distribución de los sujetos según el sexo, nivel sociocultural y edad (años, meses, días)

\begin{tabular}{cccccc}
\hline \multirow{2}{*}{ Edad } & \multicolumn{5}{c}{ Nivel sociocultural } \\
\cline { 2 - 6 } $3-3 ; 11,29$ & Sexo & Alto & Medio & Bajo & Total \\
& $\mathrm{V}$ & 7 & 6 & 8 & 43 \\
$4-4 ; 11,29$ & $\mathrm{H}$ & 8 & 7 & 7 & \\
& $\mathrm{~V}$ & 10 & 9 & 9 & 51 \\
$5-5 ; 11,29$ & $\mathrm{H}$ & 7 & 8 & 8 & \\
& $\mathrm{~V}$ & 7 & 8 & 9 & 48 \\
$6 \cdot 6 ; 11,29$ & $\mathrm{H}$ & 8 & 8 & 8 & \\
& $\mathrm{~V}$ & 10 & 10 & 10 & 56 \\
& $\mathrm{H}$ & 10 & 8 & 8 & 198 \\
\hline
\end{tabular}

\section{PROCEDIMIENTO}

Como paso previo a la administración de la prueba de evaluación de desarrollo fonológico a los sujetos, se llevó a cabo un estudio piloto con 50 sujetos, con objeto de conseguir la familiarización en transcripciones fonológicas y predecir posibles contratiempos en la pasación de la prueba.

La administración de la prueba consistió en que cada niño repitiera directamente cada una de las palabras propuestas, después de ser emitidas por el examinador. Este anotaba las producciones verbales correctas, indicándolas con una señal, y las incorrectas, indicando el error cometido.

La prueba fue administrada individualmente a cada sujeto y se grabaron en cintas magnetofónicas todas las respuestas, con objeto de realizar una posterior audición, que permitiese hacer correcciones de los datos tomados incorrectamente en la pasación.

Se procuró que el lugar donde se administrara la prueba fuese silencioso, fuera del aula y acústicamente cómodo.

El tiempo de duración del examen fonológico osciló entre los cinco y diez minutos por niño, dependiendo de la edad de los sujetos.

Una vez corregidos los datos, se calculó la fiabilidad de las correcciones entre los dos examinadores que pasaron la prueba resultando ser del $97,07 \%$. 


\section{RESULTADOS}

A continuación se exponen los resultados encontrados tanto en la producción correcta como en los distintos procesos de simplificación del habla, según el sexo, nivel sociocultural y edad de los sujetos, que serán analizados y explicados en el capítulo de las conclusiones.

\section{RESULTADOS EN CUANTO AL SEXO}

En la tabla 3 se expresan las significaciones obtenidas en cada grupo de sujetos según el sexo, en cada categoría de fonemas, en cuanto a producción correcta (C), procesos de sustitución (S) procesos relativos a la estructura de la sílaba $(R)$ y procesos de asimilación (A).

\section{a) Producción correcta}

En primer lugar, cabe mencionar que en las columnas donde se especifican las medias obtenidas en producción correcta (columnas $\mathrm{C}$ ), se aprecia que éstas son similares para ambos sexos, aunque se señala un leve aumento en las obtenidas por las niñas, respecto de los niños, en la mayoría de las distintas categorías de fonemas (excepto en las fricativas $/ \theta /, / f /, / x /$, africada y nasales). En consecuencia no se encuentran diferencias significativas en las producciones correctas de las distintas categorías de fonemas según el sexo de los sujetos, excepto en las fricativas $/ \mathrm{s} /$ y $/ \theta /(\mathrm{p}<0.10)$, líquida vibrante $/ \overline{\mathrm{r}} /$ y grupo consonántico $/ \mathrm{Ls} /(\mathrm{p}<0.01)$.

\section{b) Procesos de sustitución}

En cuanto a la producción de los procesos de sustitución cabría señalar que en las columnas donde se especifican las medias obtenidas (columna $S$ ), se aprecia que éstas son muy similares para ambos sexos, aunque contrariamente a las de producción correcta, las puntuaciones obtenidas en la mayoría de las categorías de fonemas (excepto en $/ f /, / x /$, nasales, $/ \lambda /$ ), son levemente mayores en los niños que en las niñas. Por tanto, sólo se encuentran diferencias significativas en cuanto al sexo, en los fonemas $/ \theta /(\mathrm{p}<0.10) ; / \mathrm{r} /(\mathrm{p}<0.01)$ y grupo consonántico /Ls/ $(\mathrm{p}<0.05)$.

\section{c) Procesos relativos a la estructura de la silaba}

En cuanto a la producción de los procesos relativos a la estructura de la sílaba, cabría señalar que en las columnas donde se especifican las medias obtenidas por los sujetos (columnas R), se encuentra que éstas al igual que lo que ocurría en las columnas $\mathrm{S}$ y $\mathrm{C}$, son muy similares entre sí para ambos sexos, aunque contrariamente a ellas, algo más bajas en general, encontrándose que las puntuaciones medias en la mayoría de las distintas categorías fonemáticas (excepto en $/ \mathrm{x} /$, africada y G.C. /Ls/ son levemente mayores en los niños que en las niñas. Sólo se encuentran diferencias significativas en el fonema /f/ $(p<0.10)$.

\section{d) Procesos de asimilación}

Por último, los procesos de asimilación (columnas A) apenas se encuentran en las distintas categorías de fonemas y donde aparecen es levemente superior en los niños que en las niñas. Estas diferencias no son significativas a ningún nivel y en ninguna categoría de fonemas. 
TABLA III

Medias y Coeficientes $F$ de las producciones correctas $(C)$, procesos de sustitución $(S)$, relativos a la estructura de la silaba $(R)$, de asimilación $(A)$, emitidos por los sujetos de ambos sexos, en las distintas categorias de fonemas

$\overline{\mathbf{X}}$

\begin{tabular}{|c|c|c|c|c|c|c|c|c|c|c|c|c|c|}
\hline & \multicolumn{4}{|c|}{ Niños } & \multicolumn{4}{|c|}{ Niñas } & \multicolumn{4}{|c|}{ T } \\
\hline & & C & $\mathbf{S}$ & $\mathbf{R}$ & $\mathbf{A}$ & C & $\mathbf{S}$ & $\mathbf{R}$ & A & $\mathrm{C}$ & $\mathbf{S}$ & $\mathbf{R}$ & $\mathbf{A}$ \\
\hline Oclusivas & & 11,56 & 0,35 & 0,07 & 0,03 & 11,65 & 0,23 & 0,08 & 0,03 & 0,67 & 1,41 & 0,20 & 0,01 \\
\hline \multirow{4}{*}{ 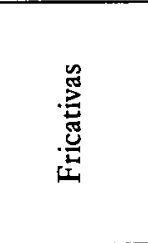 } & $/ \mathrm{s} /$ & 1,57 & 1,03 & 0,40 & - & 1,84 & 0,84 & 0,32 & - & $\begin{array}{c}3,21 \\
*\end{array}$ & 2,34 & 1,59 & - \\
\hline & $/ \theta /$ & 2,25 & 0,50 & 0,48 & - & 2,13 & 0,31 & 0,44 & - & $\begin{array}{c}3,86 \\
\star\end{array}$ & $\begin{array}{c}3,43 \\
*\end{array}$ & 0,27 & - \\
\hline & $/ f /$ & 1,87 & 0,11 & 0,03 & - & 1,86 & 0,14 & - & - & - & 0,38 & $\begin{array}{c}2,79 \\
\star\end{array}$ & - \\
\hline & $|x|$ & 1,99 & - & 0,01 & - & 1,97 & 0,01 & 0,02 & 0,01 & 1,21 & 1,10 & 0,43 & 1,10 \\
\hline Africada & & 1,94 & 0,05 & 0,01 & - & 1,92 & 0,05 & 0,02 & 0,01 & 0,36 & 0,02 & 0,43 & 1,10 \\
\hline Nasales & & 5,89 & - & 0,10 & 0,01 & 5,87 & 0,01 & 0,07 & 0,04 & 0,19 & 1,10 & 0,32 & 2,14 \\
\hline \multirow{4}{*}{ 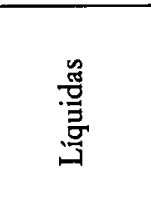 } & $M$ & 216 & 0,07 & 0,32 & $0 ; 01$ & 2,66 & 0,05 & 0,28 & - & 0,40 & 0,14 & 0,19 & 0,91 \\
\hline & $N$ & 0,02 & 1,96 & 0,02 & - & 0,01 & 1,98 & 0,01 & - & 0,25 & 0,51 & 0,25 & - \\
\hline & $/ \mathrm{r} /$ & 1,45 & 0,13 & 0,38 & 0,05 & 1,48 & 0,07 & 0,36 & 0,05 & 0,12 & 1,08 & 0,05 & 1,06 \\
\hline & $\mid \overline{\mathbf{r}} /$ & 1,24 & 0,71 & 0,02 & 0,03 & $1,58^{\circ}$ & 0,39 & 0,01 & 0,02 & $\begin{array}{l}8,23 \\
\star \star \star \\
\end{array}$ & $\begin{array}{l}7,93 \\
\star \star \star \\
\end{array}$ & 0,25 & 0,09 \\
\hline \multicolumn{2}{|l|}{ G.C.L. } & 10,23 & 1,32 & 3,76 & 0,07 & 10,89 & 1,12 & 3,20 & 0,08 & 0,86 & 0,43 & 0,76 & 0,14 \\
\hline \multicolumn{2}{|l|}{ G.C. $/ \mathrm{Ls} /$} & 0,08 & 0,91 & 0,01 & - & 0,31 & 0,67 & 0,02 & - & $\begin{array}{l}7,62 \\
\star \star \star\end{array}$ & $\begin{array}{c}4,69 \\
\star \star\end{array}$ & 0,62 & - \\
\hline \multicolumn{2}{|l|}{ O.G.C. } & 7,31 & 2,37 & 0,43 & 0,11 & 7,54 & 2,26 & 0,34 & 0,11 & 0,55 & 0,16 & 0,93 & - \\
\hline \multicolumn{2}{|l|}{ Diptongos } & 6,01 & 0,36 & 0,47 & 0,16 & 6,14 & 0,29 & 0,44 & 0,13 & 0,85 & 0,61 & 0,09 & 0,55 \\
\hline
\end{tabular}

(*) $\mathrm{p}<0,10 ;(* *) \mathrm{p}<0,05 ;(* \star *) \mathrm{p}<0,01$. 


\section{RESULTADOS EN CUANTO AL NIVEL SOCIOCULTURAL}

En la tabla 4 se expresan las significaciones obtenidas en cada categoría de fonemas según el nivel sociocultural, en cuanto a producciones correctas (C), procesos de sustitución (S), relativos a la estructura de la sílaba (R) y de asimilación (A).

\section{a) Producción correcta}

En cuanto a la producción correcta, cabe señalar que en las columnas $C$ de la tabla 4, donde se especifican las medias obtenidas en cada nivel sociocultural, se aprecia un ligero aumento en el nivel alto y medio, con respecto al bajo, en la mayoría de las distintas categorías fonemáticas (excepto en oclusivas). Estas diferencias son significativas en los fonemas laterales $/ /$ y $/ \lambda /$, y en el vibrante $/ \mathrm{r} /$, en todos los grupos consonánticos y en los diptongos.

Los niveles socioculturales que difieren entre sí, en la producción correcta de los distintos fonemas se presentan a continuación. En el fonema $/ \lambda /$ el nivel alto difiere significativamente del bajo. En los fonemas $/ / \mathrm{y} / \mathrm{r} / \mathrm{y}$ en los grupos consonánticos con líquidas, los niveles alto y medio se diferencian significativamente del bajo. Por último, hay diferencia entre los niveles alto y bajo, en los Grupos consonánticos, excepto en los formados por oclusiva más líquida y en los diptongos.

\section{b) Procesos de sustitución}

En la producción de procesos de sustitución, cabría señalar que en las columnas donde se especifican las medias obtenidas (columnas S), se aprecia que éstas son considerablemente menores que las de las columnas de producción correcta, y contrariamente, en la mayoría de las categorías de fonemas (excepto en oclusivas, africada y nasales) son mayores en el nivel sociocultural bajo, respecto al medio y bajo, y muy similares entre estos últimos aunque levemente superiores en el nivel medio (excepto en oclusivas, africada $y / r /$. Sin embargo, éstas son considerablemente menores que las de las columnas de producción co$(\mathrm{p}<0.10)$, G.L.C. $(\mathrm{p}<0.05)$ y O.G.C. $(\mathrm{p}<0.05$ y $\mathrm{p}<0.10)$.

Los niveles socioculturales que difieren entre sí en la omisión de procesos de sustitución, en las distintas categorías de fonemas, son los siguientes: en los fonemas $/ \mathrm{K}$ y $/ \mathrm{r} /$, la diferencia se aprecia entre los niveles medio y bajo, y en los grupos consonánticos entre los niveles alto y bajo.

\section{c) Procesos relativos a la estructura de la sílaba}

En las columnas donde se especifican las medias obtenidas por los sujetos en la producción de estos procesos fonológicos (columna $R$ ), se encuentra que éstas, al igual que ocurría en las de las columnas $\mathrm{S}$, son superiores a las de las columnas $\mathrm{C}$, y similares entre sí para los niveles socioculturales medio y alto en favor del medio (excepto en oclusivas, /s/, nasales /r/, O.G.C. y diptongos), y algo inferiores en los niveles alto y medio, respecto al bajo.

Estas diferencias son en general altamente significativas $(\mathrm{p}<0.05$ y $\mathrm{p}<0.01)$ en todas las categorías de fonemas, excepto en oclusivas, fricativas $/ \mathrm{s} / \mathrm{y} / \theta /$, africada y líquidas $/ \lambda / \mathrm{y} / \overline{\mathrm{r}} /$.

Los niveles socioculturales que difieren significativamente entre sí, en la emisión de estos procesos en las distintas categorías de fonemas, son los que sigue: entre los niveles alto y medio con el bajo, se encuentran diferencias en las fricativas $/ \mathrm{f} / \mathrm{y} / \mathrm{x} /$, en las líquidas $/ / \mathrm{y} / \mathrm{r} / \mathrm{y}$ en $\mathrm{G}$.C.L.; entre el alto y el bajo tan 
TABLA IV

Medias y Coeficientes $F$ de las producciones correctas $(C)$, procesos de sustitución $(S)$, relativos a la estructura de la silaba $(R)$, de asimilación $(A)$, emitidos por los sujetos de los distintos niveles socioculturales, en las distintas categorias de fonemas

\begin{tabular}{|c|c|c|c|c|c|c|c|c|c|c|c|c|c|c|c|c|c|}
\hline & \multicolumn{12}{|c|}{$\overline{\mathbf{X}}$} & \multirow{2}{*}{\multicolumn{4}{|c|}{$\mathbf{F}$}} \\
\hline & & \multicolumn{4}{|c|}{ Alto } & \multicolumn{4}{|c|}{ Medio } & \multicolumn{4}{|c|}{ Bajo } & & & & \\
\hline & & C & S & $\mathbf{R}$ & A & C & $\mathbf{S}$ & $\mathbf{R}$ & A & C & $\mathbf{S}$ & $\mathbf{R}$ & $\mathbf{A}$ & C & S . & $\mathbf{R}$ & $\mathbf{A}$ \\
\hline \multicolumn{2}{|c|}{ Oclusivas } & 11,47 & 0,41 & 0,09 & 0,03 & 11,63 & 0,25 & 0,06 & 0,06 & 11,72 & 0,21 & 0,07 & - & 1,58 & 1,70 & 0,15 & 1,29 \\
\hline \multirow{4}{*}{ 胥 } & $/ \mathrm{s} /$ & 1,82 & 0,81 & 0,37 & - & 1,67 & 1,03 & 0,30 & - & 1,60 & 0,99 & 0,42 & - & 0,76 & 1,24 & 1,00 & - \\
\hline & $/ \theta /$ & 2,22 & 0,37 & 0,41 & - & 2,09 & 0,47 & 0,44 & - & 2,07 & 0,39 & 0,54 & - & 0,59 & 0,33 & 1,09 & - \\
\hline & $/ f /$ & 1,91 & 0,09 & - & - & 1,89 & 0,11 & - & - & 1,79 & 0,16 & 0,04 & - & 1,90 & 0,81 & $\begin{array}{c}3,05 \\
* *\end{array}$ & - \\
\hline & $|x|$ & 1,99 & 0,01 & - & - & 2,00 & - & - & - & 1,96 & - & 0,44 & - & 1,75 & 0,96 & $\begin{array}{c}3,05 \\
* * \\
\end{array}$ & - \\
\hline \multicolumn{2}{|l|}{ Africada } & 1,91 & 0,07 & - & 0,01 & 1,94 & 0,05 & 0,02 & - & 1,94 & 0,03 & 0,03 & - & 0,17 & 0,48 & 1,01 & 0,96 \\
\hline \multicolumn{2}{|l|}{ Nasales } & 5,87 & 0,01 & 0,07 & 0,04 & 5,95 & - & 0,03 & 0,02 & 5,84 & - & 0,15 & 0,01 & 2,15 & 0,96 & $\begin{array}{c}3,06 \\
\star \star\end{array}$ & 0,76 \\
\hline \multirow{4}{*}{ 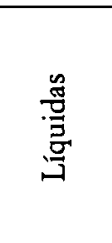 } & $N$ & 2,79 & 0,03 & 0,18 & - & 2,77 & 0,03 & 0,20 & - & 2,34 & 0,12 & 0,52 & 0,01 & $\begin{array}{c}11,64 \\
\star \star \star\end{array}$ & $\begin{array}{c}2,32 \\
\star\end{array}$ & $\begin{array}{l}9,52 \\
\star \star \star\end{array}$ & 0,98 \\
\hline & $\lambda$ & 0,04 & 1,94 & 0,01 & - & - & 1,98 & 0,02 & - & - & 1,99 & 0,01 & - & $\underset{\star \star \star}{2,98}$ & 1,45 & - & - \\
\hline & $/ \mathbf{r} /$ & 1,59 & 0,07 & 0,29 & 0,04 & 1,61 & 0,05 & 0,27 & 0,08 & 1,21 & 1,18 & 0,54 & 0,07 & $\begin{array}{l}8,41 \\
\star \star \star\end{array}$ & $\begin{array}{c}2,72 \\
*\end{array}$ & $\begin{array}{c}5,89 \\
\star \star \star\end{array}$ & 0,38 \\
\hline & $/ \overline{\mathbf{r}} /$ & 1,47 & 0,50 & 0,01 & 0,01 & 1,44 & 0,50 & 0,02 & 0,05 & 1,30 & 0,67 & 0,01 & 0,01 & 0,78 & 0,97 & - & 0,64 \\
\hline \multicolumn{2}{|l|}{ G.C.L. } & 11,84 & 0,68 & 2,59 & 0,06 & 10,95 & 1,39 & 3,05 & 0,05 & 8,85 & 1,61 & 4,84 & 0,12 & $\begin{array}{l}6,59 \\
\star * * \\
\end{array}$ & $\begin{array}{c}3,52 \\
\star \star\end{array}$ & $\begin{array}{l}4,82 \\
\star \star \star\end{array}$ & 1,00 \\
\hline \multicolumn{2}{|c|}{ G.C. /Ls/ } & 0,23 & 0,76 & 0,01 & - & 0,14 & 0,77 & 0,09 & - & 0,01 & 0,83 & 0,16 & - & $\begin{array}{c}2,78 \\
\quad *\end{array}$ & 2,07 & $\begin{array}{l}4,74 \\
\star \star \star\end{array}$ & - \\
\hline \multicolumn{2}{|l|}{ O.G.C. } & 7,85 & 1,94 & 0,34 & 0,07 & 7,47 & 2,38 & 0,27 & 0,09 & 6,93 & 2,66 & 0,55 & 0,15 & $\begin{array}{c}3,19 \\
\star *\end{array}$ & $\begin{array}{c}2,28 \\
*\end{array}$ & $\begin{array}{c}3,05 \\
\star \star\end{array}$ & 0,98 \\
\hline \multicolumn{2}{|c|}{ Diptongos } & 6,15 & 0,22 & 0,51 & 0,12 & 6,25 & 0,36 & 0,27 & 0,13 & 5,82 & 0,40 & 0,58 & 0,19 & $\begin{array}{c}3,60 \\
* *\end{array}$ & 2,05 & $\begin{array}{c}3,95 \\
\star \star\end{array}$ & 0,95 \\
\hline
\end{tabular}

$(*) \mathrm{p}<0,10 ;(* *) \mathrm{p}<0,05 ;(* * *) \mathrm{p}<0,01$ 
sólo se encuentran diferencias en el grupo consonántico /Ls/; y por último entre el nivel alto y medio sólo en los diptongos.

\section{d) Procesos de asimilación}

En los procesos de asimilación (columna A), las medias obtenidas en los tres niveles socioculturales, en la mayoría de los fonemas son nulas o muy bajas, no encontrándose diferencias significativas según el nivel sociocultural de los sujetos.

\section{RESULTADOS EN CUANTO A LA EDAD}

En la tabla 5 se recogen las significaciones obtenidas en cada categoría de fonemas y distintos tipos de respuesta (articulación correcta $-\mathrm{C}$, procesos de sustitución $-S$, relativos a la estructura de la sílaba $-R$, y asimilaciones $-A$ ), en cuanto a la edad de los sujetos.

\section{a) Producción correcta}

Cabría señalar que en las columnas donde se especifican las medias obtenidas en producción fonemática correcta (columna $\mathrm{C}$ ), se aprecia un aumento progresivo con el aumento de la edad, en todas las categorías de fonemas, excepto en el $/ \lambda /$, donde no se consiguen puntuaciones altas ni a los seis años. Por otra parte, hay algunos fonemas donde ya a los tres años las medias alcanzan casi valores máximos, como son el caso de oclusivas, fricativas $/ f / y / x / y$ nasales. De esta forma, se encontraron altas diferencias significativas $(P<0.01)$, entre edades en la producción fonemática correcta de todos los fonemas, excepto en $/ \mathrm{x} /$.

Los grupos de edad que difieren significativamente entre sí, con más frecuencia, en cuanto a la producción correcta de fonemas se refiere, son los siguientes. El grupo de tres y cuatro años con el de seis, excepto en el fonema $/ \mathrm{x} /$ y $/ \theta /$ para 3 y 6 años y el $/ x / y / \lambda /$ para 4 y 6 años. A continuación le sigue el grupo de tres años con el de 5 , cuyas diferencias se reflejan en la producción de $/ \mathrm{s} /, / \mathrm{f} /$, nasales, $/ \lambda /, / \mathrm{r} /, / \overline{\mathrm{r}} /$, y G.C.L. Seguidamente se encuentran los grupos de 5 y 6 años, donde las diferencias se presentan en todos los fonemas, excepto en oclusivas, africada, nasales, $/ \mathrm{f} / \mathrm{y} / \mathrm{x} /$. Los grupos de 4 y 5 años se diferencian en los fonemas $/ \mathrm{s} /, / \mathrm{f} /, / \lambda /, / \overline{\mathrm{r}} /$, africada y grupos consonánticos. Por último, los grupos de 3 y 4 años se diferencian en oclusivas, /s/, diptongos y O.G.C.

\section{b) Procesos de simplificación}

En cuanto a la producción de procesos fonológicos de simplificación cabría señalar, que en las columnas donde se especifican (columnas $S, R, A$ ) se aprecia que éstas son considerablemente menores que las de las columnas de producción fonemática correcta, y contrariamente a ellas, en la mayoría de los fonemas (excepto en $/ \lambda$ / para los procesos de sustitución, y en $/ \mathrm{Ls} /$ para los procesos relativos a la estructura de sílaba) van desapareciendo con el aumento de la edad. Hay algunos fonemas donde ya a los tres años apenas aparecen simplificaciones como son el caso de las sustituciones en oclusivas, africada, nasales y $/ /$; y casi nulas en los procesos relativos a la estructura de la sillaba y de asimilación, en general. Sin embargo, hay otras categorias de fonemas que incluso a los seis años presentan puntuaciones altas en estos procesos, como las sustituciones en el fonema $/ \lambda /$ y O.G.C. y los relativos a la estructura de la sílaba en los G.C.L. 


\section{TABLA V}

Medias y Coeficientes $F$ de las producciones correctas $(C)$, procesos de sustitución $(S)$, relativos a la estructura de la sillaba $(R)$, de asimilación $(A)$, emitidos por los sujetos de distintos niveles de edad, en las distintas categorias de fonemas

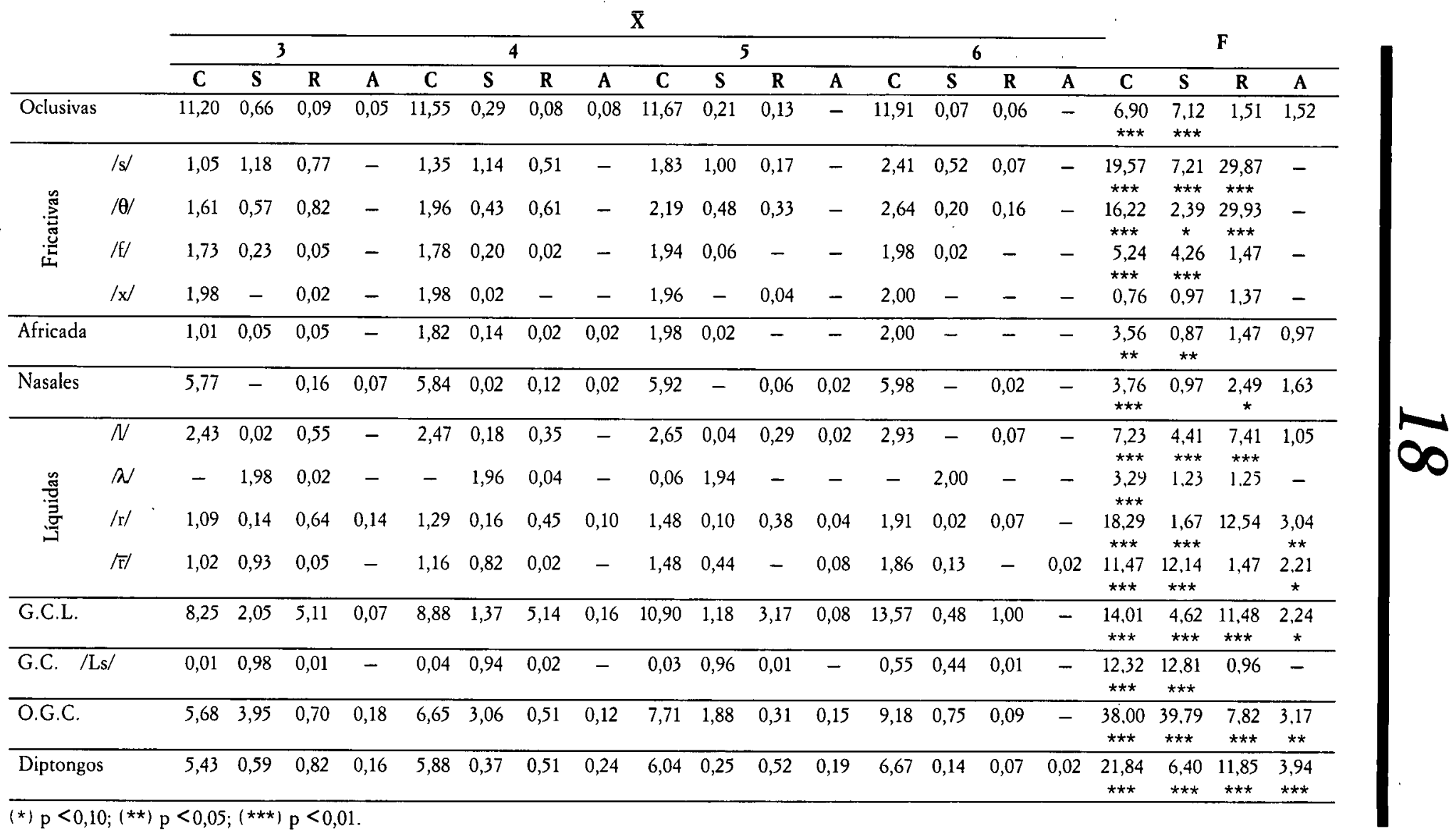


Por otra parte, cabe señalar que los procesos de simplificación más comunes en la mayoría de los fonemas, en todas las edades son los de sustitución, seguidos de los relativos a la estructura de la silaba y por último los de asimilación, que apenas se presentan.

De esta forma, los procesos de simplificación en los que existen un mayor número de fonemas en los que se encuentran diferencias significativas respecto a la edad de los sujetos, son los de sustitución (en oclusivas, /s/, / $/$ /, /f/, africada, $/ /, / \overline{\mathbf{r}} /$, G.C.L., /Ls/, O.G.C. y diptongos) y los relativos a la estructura de la silaba $(/ \mathrm{s} /, / \theta /$, nasales, $/ \mathrm{N}, / \mathrm{r} /$, grupos consonánticos y diptongos). Los procesos de asimilación sólo presentan diferencias en cuanto a la edad de los sujetos en los fonemas $/ \mathrm{r} /, / \overline{\mathrm{r}} /$, G.C.L. y O.G.C y diptongos.

Por último, en la tabla 6 quedan expresados los grupos de edad que difieren significativamente entre sí en la producción de los procesos de simplificación del habla en los distintos fonemas en que aparecen. Los pares de grupos más numerosos son los grupos de 3 y 4 con el de 6 años. Les sigue el de 3 con el de 5 , el de 5 con el de 6, el de 4 con el de 5 y el de 3 con el de cuatro años.

\section{TABLA VI}

Fonemas en los que los grupos de edad son diferentes dos a dos, en cada uno de los procesos de simplificación

\begin{tabular}{|c|c|c|c|}
\hline & \multicolumn{3}{|c|}{ Procesos de simplificación del habla } \\
\hline & Sustituciones & R. estructura silaba & Asimilac. \\
\hline $3-4$ & oclus., l, OGC, dipt. & s, $\theta$, dipt. & ---- \\
\hline $3-5$ & oclus., $\mathrm{f}, \overline{\mathrm{r}}, \mathrm{OGC}$, dipt. & $\mathrm{s}, \theta, \mathrm{l}, \mathrm{r}, \mathrm{GCL}, \mathrm{OGC}$, dipt. & $\overline{\mathrm{r}}$ \\
\hline $3-6$ & $\begin{array}{l}\text { oclus., s, } \theta, \mathrm{f}, \overline{\mathrm{r}}, \mathrm{GCL}, \mathrm{Ls}, \\
\text { OGC, dipt. }\end{array}$ & $\begin{array}{l}\text { s, } \theta \text {, nasal, l, r, GCL, OGC, } \\
\text { dipt. }\end{array}$ & $\begin{array}{l}\text { nasal, r, OGC, } \\
\text { dipt. }\end{array}$ \\
\hline $4-5$ & afric, $1, \overrightarrow{\mathbf{r}}, \mathrm{OGC}$ & $\mathrm{s}, \theta, \mathrm{GCL}$ & $\overline{\mathrm{r}}$ \\
\hline $4 \cdot 6$ & $\begin{array}{l}\text { s, f, afric, l, } \bar{r} . \mathrm{GCL}, \mathrm{Ls}, \\
\text { OGC, dipt. }\end{array}$ & $\mathrm{s}, \theta, 1, \mathrm{r}, \mathrm{GCL}, \mathrm{OGC}$, dipt. & GCL, dipt. \\
\hline $5-6$ & $s, \bar{r}, L s$, OGC & 1, r, GCL, dipt. & OGC, dipt. \\
\hline
\end{tabular}

\section{DISCUSION Y CONCLUSIONES}

En general, cabría decir que se han encontrado diferencias significativas en las producciones fonológicas de los sujetos tanto en cuanto a la edad como al nivel sociocultural, mientras que no se han encontrado en cuanto al sexo.

A continuación se realizará un análisis particular de cada uno de los resultados encontrados, con respecto a las tres variables estudiadas en la evaluación del desarrollo fonológico.

En cuanto al sexo de los sujetos, aunque las medias en producción correcta son levemente mayores y en procesos de simplificación levemente inferiores en las niñas respecto a los niños, apenas se han encontrado diferencias significativas entre las producciones fonemáticas de ambos. Estas sólo aparecen en la articulación correcta de los fonemas, $/ \mathrm{s} /, / \theta /, / \overline{\mathbf{r}} /, / \mathrm{Ls} /$, en las sustituciones de $/ \theta /$, $/ \overline{\mathrm{r}} /, / \mathrm{Ls} /$, y las relativas a la estructura de la sílaba del fonema /f/. En la mayoría de los estudios realizados sobre el desarrollo del lenguaje (Dale, 1980), aparecen en escasas ocasiones significaciones con respecto al sexo. En el presente estudio 
ocurre que apenas existen diferencias significativas y donde se encuentran, podría pensarse que más que deberse a que los niños y las niñas siguen patrones evolutivos diferentes, se deba a la existencia de una mayor o menor tendencia de ambos a cometer errores dialectales (Alvar, 1973; González, 1987, 1989), ya que los fonemas en los que aparecen las diferencias presentan una alta frecuencia de seseos, ceceos, aspiraciones, etc. Por tanto, se podría concluir que los niños y las niñas de la Provincia de Málaga no presentan distinta capacidad de representación fonemática en su propio desarrollo evolutivo. Sin embargo, sería interesante analizar en futuras investigaciones, a qué podría ser debido esta tendencia a cometer errores dialectales y de qué manera influye en la adquisición fonológica.

En cuanto al nivel sociocultural de los sujetos, en la producción correcta se han encontrado diferencias significativas en los fonemas $/ / /, r /, / 2$, grupos consonánticos y diptongos. En los procesos de sustitución se aprecian en $/ \mathrm{l} / \mathrm{h} /$, G.C.L. y O.G.C. En los procesos relativos a la sílaba se han encontrado en todas las categorías de fonemas, excepto en oclusivas, fricativas $/ \mathrm{s} / \mathrm{y} / \theta /$, africada y líquida $/ \lambda /$ y $/ \overline{\mathrm{r}} /$. Estos fonemas en los que hay diferencias significativas, son fonemas considerados de más difícil pronunciación, y posteriores en la adquisición fonológica del habla castellana (Melgar, 1976; Serra, 1979; Bosch, 1982). Por otra parte, las medias obtenidas en general en la mayoría de los fonemas son superiores en producción correcta en los niveles alto y medio con respecto al bajo, e inversamente inferiores en los procesos de simplificación fonológica en el nivel sociocultural bajo, respecto a los niveles alto y medio. Es decir, que existen diferencias significativas en cuanto a los niveles socioculturales y desarrollo fonológico y que aquéllas se presentan a favor de los niveles alto y medio. En opinión de Dale (1980), la clase social en sí misma puede ser difícilmente la que ocasione estas diferencias en el desarrollo del lenguaje. Sin embargo, aunque muy ambiguamente, variables correlacionadas con el nivel social, como son el lenguaje que oyen los niños o la forma de educación que reciben, podrían influir en el desarrollo de los sujetos. Si un niño del nivel sociocultural bajo, que pronuncia mal un fonema, no es corregido por los adultos que le rodean, o incluso el modelo al que imita tampoco pronuncia bien dichos fonemas, difícilmente podríamos esperar que dicho niño evolucione en el aprendizaje y adquisición de su lengua. Si estos sujetos no son capaces de producir correctamente algunos de los fonemas del castellano, después de emisiones adultas, es porque el ambiente lingüístico y educativo circundante no le ha provisto de la posibilidad de desarrollar, a un ritmo adecuado, su capacidad de representación fonológica. Ciertamente estos niños en ambientes adecuados presentarán las mismas medias en producción correcta que los niños pertenecientes a otros niveles socioculturales. Por tanto, estas diferencias más que debido a un retraso madurativo se debe a una desviación de tipo sociocultural, influyendo este último en la adquisición fonológica de los sujetos.

En cuanto a la edad de los sujetos, en producción correcta se han obtenido diferencias significativas en todas las categorías de fonemas, excepto en $/ \mathrm{x} /$, donde ya a los tres años la pronunciación correcta alcanza su valor máximo. De acuerdo con la teoría psicolingüística, a los tres años, la representación fonológica del sonido fricativo $/ x /$ es posible, no encontrándose variaciones significativas en el resto de las edades. Tampoco hay que olvidar que dicho fonema tiene características propias que lo hacen fácilmente pronunciable y es quizá por ello por lo que su representación y producción correcta sea primaria. En el mismo caso es- 
tán los fonemas oclusivos, fricativo /f/, africada y nasales, aunque en ellos no se consiguen puntuaciones tan elevadas a los tres años como en $/ \mathrm{x} /$, y se encontraron diferencias significativas según la edad de los sujetos. Por otra parte, en la producción correcta del fonema $/ \lambda /$, no se consiguen puntuaciones altas ni a los seis años, presentándose un alto porcentaje de simplificaciones debido a la influencia de variantes dialectales generalizadas, como es el yeísmo. El hecho de que un niño de seis años no pronuncie correctamente dicho fonema, y lo sustituya por otro, no quiere decir que no lo perciba y organice según sus características. De esta forma, la influencia del dialecto, no necesariamente tendría que retrasar el desarrollo evolutivo del niño, aunque sí podría enmascararlo, pareciendo así que siguen patrones fonológicos más lentos. Sería necesario en el futuro un estudio de las habilidades discriminativas auditivas de los sujetos para confirmar tan evidentes resultados. De igual forma que el fonema $/ \lambda /$, se encuentran los fonemas $/ \mathrm{s} /, / \theta /$, y algunos de los grupos consonánticos, donde se encuentran procesos dialectales andaluces como seseo, ceceo, aspiraciones, lateralización de vibrantes, omisiones de $/ 1 /, / r / y / s /$, en posición final de palabra, etc. (Zamora, 1979).

En cuanto a los procesos de simplificación del habla, según la edad de los sujetos, y de acuerdo con la mayoría de los autores que han trabajado en esta línea (Ingram, 1976; Bosch, 1982; Dyson y Paden, 1983; Haelsig y Madison, 1986; Hernández Pina, 1984), se han encontrado un descenso progresivo en todos ellos con la edad, en el valor de las medias obtenidas en la mayoría de los fonemas (excepto en $/ \lambda /$ y $/ \mathrm{Ls} /$, ambos sujetos a la influencia de variables dialectales). Esto implica, que a medida que avanza el desarrollo normal del niño, su habla simplificada tiende a desaparecer hasta que consigue caracterizarse como la del modelo adulto andaluz (Ingram, 1976, 1979). Cabe mencionar que los procesos de simplificación que se han encontrado más frecuentemente en las categorías de fonemas en toda las edades, son los de sustitución, seguidos de los relativos a la silaba y los de asimilación. Esto hace pensar que si a los trece años los procesos de asimilación y relativos a la sílaba son ya poco frecuentes es porque los sujetos ya poseen cierto nivel de desarrollo fonológico (Hernández Pina, 1984). Esto viene además confirmado cuando se encuentra a esta edad fonemas (oclusivas, fricativa $/ \mathrm{x} / \mathrm{y} / \mathrm{f} /$, africada, nasales) que su producción correcta es prácticamente máxima. Por otra parte, cabe señalar que el hecho de que aún a los seis años existan procesos de sustitución en algunos fonemas $/ \lambda /, / \mathrm{s} /$, $/ \theta /$, O.G.C., G.C.L.) es debido, como se dijo anteriormente, a la influencia de variables dialectales de la lengua andaluza, ya que las aspiraciones, sẹseos, ceceos... son procesos de sustitución y frecuentes en el habla de Andalucía (González, 1989).

Por último, puede apreciarse que las diferencias significativas, tanto en producción correcta como en procesos de simplificación del habla, son menos frecuentes entre los grupos de edades consecutivas. Por el contrario, donde más frecuentemente se encuentran es entre los grupos de tres y cuatro años con el de seis, y el de tres con el de cinco años. Estos resultados hacen pensar que a los cuatro años aparece un cambio en la adquisición fonológica, llegando a completarse casi totalmente el sistema fonológico infantil, a los seis años. Se aprecia que en los fonemas de fácil adquisición no se encuentran diferencias entre edades tempranas consecutivas, mientras que sí aparecen esas diferencias en fonemas de más difícil pronunciación, como son las fricativas, líquidas y grupos consonánticos (Haelsig y Madison, 1986). 
Como conclusión final respecto a la edad de los sujetos y su desarrollo fonológico, habría que decir que son significativas las diferencias en cuanto a las producciones fonológicas, como era de esperar. A medida que un niño evoluciona se constituye su sistema de representación fonológico, y cada vez se acerca más al modelo adulto que le rodea. No hay que olvidar que el modelo andaluz de los niños malagueños con los que se ha trabajado, se caracteriza por variables dialectales claras que determinan, si no ya la capacidad de percepción y organización fonológica, sí su producción en cuanto al habla castellana. Esto no implica un retraso en el desarrollo, ni necesariamente patrones evolutivos distintos a los establecidos para los demás niños de habla castellana. En primer lugar, el hecho de que un niño malagueño no produzca correctamente los fonemas sujetos a errores dialectales, no necesariamente quiere decir que no discrimine o perciba su diferencia respecto a otros fonemas, y mucho menos que no posea capacidad de representación fonológica adecuada. En segundo lugar, se ha encontrado aparentemente la misma secuencia en la adquisición fonológica que en otros trabajos realizados con sujetos castellanoparlantes (Bosch, 1982), sólo que algo enmascarado por la influencia dialectal anteriormente citada. Así los fonemas anteriormente adquiridos son los oclusivos, africada, nasales, fricativas / $f$ / y $/ \mathrm{x} /$, mientras que los más tardíos son los frictivos $/ \mathrm{s} / \mathrm{y} / \theta /$, líquidos, grupos consonánticos y diptongos.

\section{Notas}

1 Los grupos consonánticos formados por oclusiva más líquida, están representados en este trabajo con las siglas G.C.L.; los grupos formados por consonante más oclusiva, están representados por O.G.C., a excepción del grupo /Ls/, que se representa como G.C. $/ L s /$.

\section{Referencias}

Alegria, J. (1985). «Por un enfoque psicolingüistico del aprendizaje de la lectura y sus dificultades». Infancia y aprendizaje, 29, 79-75.

Alvar, M. y cols. (1969).. Curso de fonética y fonologia, Madrid, C.S.I.C.

- (1973). Atlas lingüistico-etnológico de Andalucia, tomo VI, Univ. de Granada, C.S.I.C.

Bandura, A. (1982), Teoría del aprendizaje social, Madrid, Espasa-Calpe.

BosCH, L. (1982). Prueba para la evaluación del desarrollo fonológico en niños de edades comprendidas entre 3 y 7, 11 años. Memoria de Licenciatura, Univ. de Barcelona.

- (1983). «El desarrollo fonológico infantil: una prueba para su evaluación». En: Siguan, M. (ed): Estudios sobre Psicologia del lenguaje infantil, Madrid, Pirámide.

Canellada, M. J. y Kuhlmann, J. (1987). Pronunciación del español, Madrid, Castalia.

Clark, H. H. y Clark, E.V. (1977). Psychology and language. The study of Development psycholinguistics. Nueva York, Harcourt Brace Jovanovich, Inc.

DALE, P.S. (1980). Desarrollo del lenguaje: un enfoque psicolingüistico, México, Trillas.

DYSON, T.D. y PADEN, P.E. (1983). «Some phonological adquisition strategies used by two-yearsolds». Journal of Childhood Communications Disorder 7 vols, 1, 6-18.

GAmmon, S. C. y COOPER, A. J. (1984). «Patters of early lexical and phonological development". Journal of child language, 2, 247-271.

GARBER, N. (1986). «A phonological analysis classification for use with traditional articulation tests. Language, Speech and Hearing Services in Scbools, 7.

GARN.NUMM, G.P. (1986). «Phonological processes and conventional articulation tests: considerations for analysism. Language Speech and Hearing Services in Schools, 17, 244-252.

GONZALEZ VALENZUELA, M.J. (1987). Estudio sobre la evaluación del desarrollo fonológico realizado con niños de la provincia de Málaga. Memoria de Licenciatura. Universidad de Málaga.

- (1989). «Importancia de las variables psicosociolingüísticas en la evaluación del desarrollo fonológico de niños malagueños». Rev. Logopedia, Foniatría y Audiología, Vol. XI, núm. 1. 